\title{
Significance of prohibitin domain family in tumorigenesis and its implication in cancer diagnosis and treatment
}

\author{
Jie Yang ${ }^{1}$, Bin $\mathrm{Li}^{1}$ and Qing-Yu He (1)
}

\begin{abstract}
Prohibitin (PHB) was originally isolated and characterized as an anti-proliferative gene in rat liver. The evolutionarily conserved PHB gene encodes two human protein isoforms with molecular weights of $\sim 33 \mathrm{kDa}$, PHB1 and PHB2. PHB1 and PHB2 belong to the prohibitin domain family, and both are widely distributed in different cellular compartments such as the mitochondria, nucleus, and cell membrane. Most studies have confirmed differential expression of PHB1 and PHB2 in cancers compared to corresponding normal tissues. Furthermore, studies verified that PHB1 and PHB2 are involved in the biological processes of tumorigenesis, including cancer cell proliferation, apoptosis, and metastasis. Two small molecule inhibitors, Rocaglamide (RocA) and fluorizoline, derived from medicinal plants, were demonstrated to interact directly with PHB1 and thus inhibit the interaction of PHB with Raf-1, impeding Raf-1/ERK signaling cascades and significantly suppressing cancer cell metastasis. In addition, a short peptide ERAP and a natural product xanthohumol were shown to target PHB2 directly and prohibit cancer progression in estrogen-dependent cancers. As more efficient biomarkers and targets are urgently needed for cancer diagnosis and treatment, here we summarize the functional role of prohibitin domain family proteins, focusing on PHB1 and PHB2 in tumorigenesis and cancer development, with the expectation that targeting the prohibitin domain family will offer more clues for cancer therapy.
\end{abstract}

\section{Facts}

- PHB1 and PHB2 are widely distributed in cells and correlate with diverse diseases.

- PHB1 and PHB2 are involved in multiple biological processes in tumorigenesis like proliferation, metastasis and apoptosis.

- PHB1 and PHB2 are regulated by transcriptional regulation, post-transcriptional regulation and protein modification in cancer cells.

- Several small molecular inhibitors targeting PHB1

Correspondence: Bin Li (libin2015@jnu.edu.cn) or Q-Y. He (tqyhe@jnu.edu.cn) ${ }^{1}$ Key Laboratory of Functional Protein Research of Guangdong Higher Education Institutes, Institute of Life and Health Engineering, College of Life Science and Technology, Jinan University, 510632 Guangzhou, China Edited by P. Pinton and PHB2 have significant impacts on cancer therapy.

\section{Questions}

- Why PHB1 exerts controversial impacts on cell proliferation in different cancers?

- Which transcription factors regulate PHB1 expression in cancer cells?

- What are the mechanisms on regulation of PHB2 in cancer cells?

- Can PHB1 or PHB2 inhibitors enhance the treatment efficiency of chemotherapeutic drugs?

\section{Introduction}

The prohibitin $(P H B)$ gene was originally isolated and characterized as a candidate anti-proliferative gene in rat 
liver cells ${ }^{1}$, and the homologous human gene maps to chromosome $17 \mathrm{q} 21-21^{2-4}$, encoding two $\sim 33 \mathrm{KD}$ proteins, PHB1 and $\mathrm{PHB}_{2}^{5}$. PHB1 and PHB2 belong to the evolutionarily conserved band-7 family, or prohibitin domain family ${ }^{5}$. Both PHB1 and PHB2 are widely distributed in different cellular compartments including the mitochondria, nucleus and cell membrane, with diverse biological functions. PHB1 located in the inner mitochondrial membrane interacts with PHB2 to stabilize the mitochondria. As mitochondria are the main energy machines for biological activities in cells, expression changes in PHB1 or PHB2 in the mitochondria always induce mitochondria-related processes such as apoptosis ${ }^{6}$, 7.

PHB1 protein located in the cell membrane functions as viral or bacterial receptors to facilitate the entry of these microorganisms into host cells ${ }^{8}$. Meanwhile, PHB1 located in the lipid raft of the cell membrane interacts with and activates Raf- 1 , an evolutionarily conserved oncogene that activates ERK and promotes cancer development ${ }^{9}$. In addition, membrane-localized PHB2 also promotes cancer cell migration ${ }^{10}$. Moreover, PHB1 located in the nucleus binds to several transcription factors, such as p53, E2F, and $\mathrm{pRb}$. Early studies claimed that PHB1 accumulates in the nucleus to induce cell cycle arrest and inhibit cell proliferation; however, this theory was challenged in recent years ${ }^{5,11,12}$. Nuclear PHB2 seems to be primarily involved in centromeric cohesion protection and promotion of cell growth ${ }^{13}$.

PHB1 and PHB2 are also involved in many biological processes related to tumorigenesis. Studies have found that overexpression of PHB1 and PHB2 results in cancer cell metastasis and apoptosis ${ }^{10,}{ }^{14-16}$. Interestingly, although PHB1 was first identified as an anti-proliferative protein, the role of PHB1 in cancer cell proliferation remains controversial $^{17,18}$. As PHB1 and PHB2 have been shown to be differentially expressed in multiple cancers, PHB1 and PHB2 are potentially useful as new biomarkers and targets in cancer diagnosis and treatment.

Natural products provide an invaluable source of medicinal leads, presenting a significant impact on drug development. RocA is isolated from Aglaia species (Meliaceae), and fluorizoline is synthetized based on natural products from medicinal plants ${ }^{19-21}$. Both RocA and fluorizoline have been reported to interact with PHB1 directly and disrupt the interaction of PHB1 and Raf-1, therefore inhibits the activation of Raf-1/ERK signaling cascades and suppresss cancer cell growth and metas$\operatorname{tasis}{ }^{14,22}$. RocA was also shown to significantly suppress cancer development in some drug-resistant cells ${ }^{23}$. Moreover, ERAP, a short synthetic peptide, and xanthohumol, a natural product from medical plants, were demonstrated to suppress cancer cell proliferation by targeting PHB2 ${ }^{24,} 25$, indicating that drugs targeting PHB1 and PHB2 may be a promising strategy for cancer treatment.

Although there have been substantial advances in our understanding on the mechanisms of tumorigenesis, efficient remedies for diagnosis and treatment of cancer are still lacking. Considering the special localization and significant roles of prohibitin domain family proteins in cancer, the value of PHB1 and PHB2 in cancer treatment warrants further detailed study. Here, we summarize the current understanding on the functional role of PHB1 and PHB2 in biological processes, particularly tumorigenesis.

\section{Location and function of PHB1 and PHB2}

The microenvironment in which proteins reside offers the perfect conditions to exert their function, therefore, localization has a large impact on protein function. According to the literature, both PHB1 and PHB2 are ubiquitously expressed, either in circulating form or in multiple cellular compartments, including the mitochondria, nucleus and plasma membrane ${ }^{6,11,26,27 .}$

\section{PHB1 and PHB2 locate in the inner mitochondrial membrane}

PHB1 located in the inner mitochondrial membrane maintains mitochondrial stability by interacting with PHB2 to form a PHB1/PHB2 complex when mitochondria encounter metabolic stress ${ }^{6,28-30}$. This process modulates the balance between mitochondrial fusion and fission events $^{31,32}$, thus maintaining a healthy mitochondrial network that protects cells from mitochondria-related apoptosis ${ }^{7}, 33,34$. Former studies reported that loss of PHB1 and PHB2 in podocytes disrupts the activation of mTORC1 and inhibits kidney filtration ${ }^{31,35}$. Levels of mitochondrial PHB1 are significantly decreased in the olfactory bulb, indicating that PHB1 is a driver of olfactory neurodegeneration in intermediate and advanced Alzheimer's disease stages ${ }^{36}$. Another study demonstrated that loss of PHB2 from the mitochondrial membrane leads to tau hyperphosphorylation and neurodegeneration ${ }^{37}$.

Interestingly, experiments performed in transgenic mice illustrated that neuronal expression of mitochondrial PHB1 confers profound neuroprotection ${ }^{38,}$ 39. A proteomics comparison between the substantia nigra $(\mathrm{SN})$ and ventral tegmental area (VTA) dopaminergic neurons also demonstrated neuroprotection of mitochondrial PHB1 in Parkinson's disease ${ }^{40}$. Moreover, PHB1 in the mitochondrial membrane is also involved in the regulation of sperm motility as shown by alterations in mitochondrial membrane potential in infertile men with poor sperm quality ${ }^{41}$. A recent study on PHB2 located in the inner mitochondrial membrane verified that PHB2 acts as a crucial mitophagy receptor involved in targeting mitochondria for autophagic degradation. Briefly, PHB2 was shown to bind the autophagosomal membrane-associated 
protein LC3 through an LC3-interacting region domain upon mitochondrial depolarization and proteasomedependent outer membrane rupture, thus inducing eukaryotic mitophagy ${ }^{42}$.

\section{PHB1 and PHB2 locate in nucleus}

Nuclear PHB1 modulates transcriptional activity directly through the interactions with various transcription factors, or indirectly through the interactions with chromatin remodeling proteins ${ }^{5,11,12,43}$. The level of nuclear PHB1 can be down-regulated upon androgen treatment in cancer cells, indicating that PHB1 has a regulatory role in cell cycle progression ${ }^{44}$. In prostate cancer cells, PHB1 interacts with and suppresses E2F1 expression, repressing E2F-mediated transcription and inducing cell cycle arrest ${ }^{41,45}$. PHB1 in the nucleus also functions as a potent transcriptional corepressor for estrogen receptor $\alpha(E R \alpha)$ to abrogate cell proliferation ${ }^{46}$. Moreover, the investigation on paclitaxel resistance in cancer cells demonstrated that ER $\alpha$ promotes PHB1 mitochondrial-to-nuclear translocation to regulate estrogen-dependent paclitaxel resistance ${ }^{47}$.

In leukemic cells, PHB1 is strongly expressed in the nucleus and is a useful biomarker for the identification of leukemia subtypes ${ }^{48}$. PHB2 in the nucleus is phosphorylated by AKT at Ser-91, acts as a putative nuclear substrate of AKT and induces the differentiation of acute promyelocytic leukemia cells ${ }^{49}$, 50 . Other studies demonstrated that PHB2 is essential for protecting centromeric cohesion in the regulation of sister-chromatid cohesion during mitosis, indicating that PHB2 is necessary for proper mitotic progression ${ }^{13}$.

\section{PHB1 and PHB2 locate in cell membrane}

Some PHB1 proteins have been reported to interact with low density detergent-insoluble lipid raft domains in the plasma membrane ${ }^{26,51}$, acting as transmembrane adapters to activate downstream signals ${ }^{14,52,53}$. Previous studies also identified that PHB1 on plasma membrane acts as a viral receptor protein to facilitate virus entry into host cells 8 , 54, 55. In addition, studies found that expression level of PHB1 on T cell surfaces is significantly upregulated when $\mathrm{T}$ cells are activated ${ }^{56}$. Interestingly, another study claimed that interaction of PHB1 and Vi capsular polysaccharide (Vi) on $\mathrm{T}$ cell plasma membrane is crucial to inhibit $\mathrm{T}$ cell activation ${ }^{57,58}$. Moreover, PHB1 located on $S$. typhi-host cell plasma membrane interacts with Vi to down-regulate early inflammatory responses, indicating that PHB1 contributes to the pathogenesis of typhoid fever ${ }^{58}$. In pancreatic cancer tissue, the expression level of PHB1 on plasma membrane was found to be higher than that in normal tissues, indicating an important role of PHB in tumorigenesis ${ }^{59}$. In recent studies, PHB1 located on the platelet membrane was demonstrated to be involved in PAR1-mediated human platelet aggregation ${ }^{60,61}$, while PHB2 located in rhabdomyosarcoma (RMS) cell membrane was reported to act as a regulator in IGFBP-6-induced RMS cell migration through its interaction with insulin-like growth factor (IGF)-binding protein (IGFBP)- $6^{10}$.

Collectively, PHB1 and PHB2 located in different cellular compartments function in different biological processes, which are involved in multiple diseases (Fig. 1). The functional role of PHB1 and PHB2 in tumorigenesis and the mechanism involved requires further investigation for potential implication of prohibitin domain family proteins in tumor diagnosis and treatment.

\section{Role of PHB1 and PHB2 in tumorigenesis}

Data from The Cancer Genome Atlas (TCGA) and The Human Protein Atlas show that PHB1 and PHB2 are widely expressed in diverse cancers, at both mRNA and protein levels (Fig. 2, Fig. 3) ${ }^{62}$. A lot of evidence demonstrated that PHB1 and PHB2 are involved in biological processes of cancer development, such as proliferation, apoptosis, and metastasis.

\section{Roles of PHB1 and PHB2 in cancer cell proliferation}

Since the original identification of PHB1 as a proliferation suppressor in rat liver cells ${ }^{1}$, the effect of PHB1 on cancer proliferation has been studied. Some studies found that PHB1 inhibits breast cancer cell proliferation by up-regulating androgen receptor (AR) expression in estrogen receptor (ER)-positive breast cancer cells ${ }^{15}$, 63 . PHB1 also inhibits proliferation of human osteosarcoma MG-63 cells by interacting with tumor suppressors such as $\mathrm{p} 53^{64}$. In glioma cells, PHB1 expression is down regulated by miR-26a, which interferes with the regulation of PHB1 on expression levels of HIF-1 and VEGF, as well as tumor growth ${ }^{65,66}$. Moreover, PHB1 is significantly down regulated in nasopharyngeal carcinoma and hepatocellular carcinoma ${ }^{17,67}$, indicating that PHB1 may be a promising diagnostic biomarker in these cancers.

However, PHB1 exhibits opposing functions in other cancer types. For example, down-regulation of PHB1 inhibits cancer cell proliferation by inducing G1-G0 arrest in esophageal squamous cell carcinoma $(\mathrm{ESCC})^{18}$. Overexpression of PHB1 was verified in gallbladder cancer tissues, and further studies identified that PHB1 promotes cancer cell proliferation by activating ERK and downstream signaling pathways ${ }^{68}$. Similarly, PHB1 was also demonstrated to be overexpressed in bladder cancer tissues; mechanistically, AKT phosphorylates PHB1 at T258, inducing mitochondrial localization of PHB1 and leading to cancer cell proliferation ${ }^{69,}{ }^{70}$. Moreover, PHB1 is overexpressed in ovarian epithelial tumors and induces cell proliferation ${ }^{71,} 72$. In estrogen-dependent cancers such as breast cancer, investigators reported that BIG3, a 


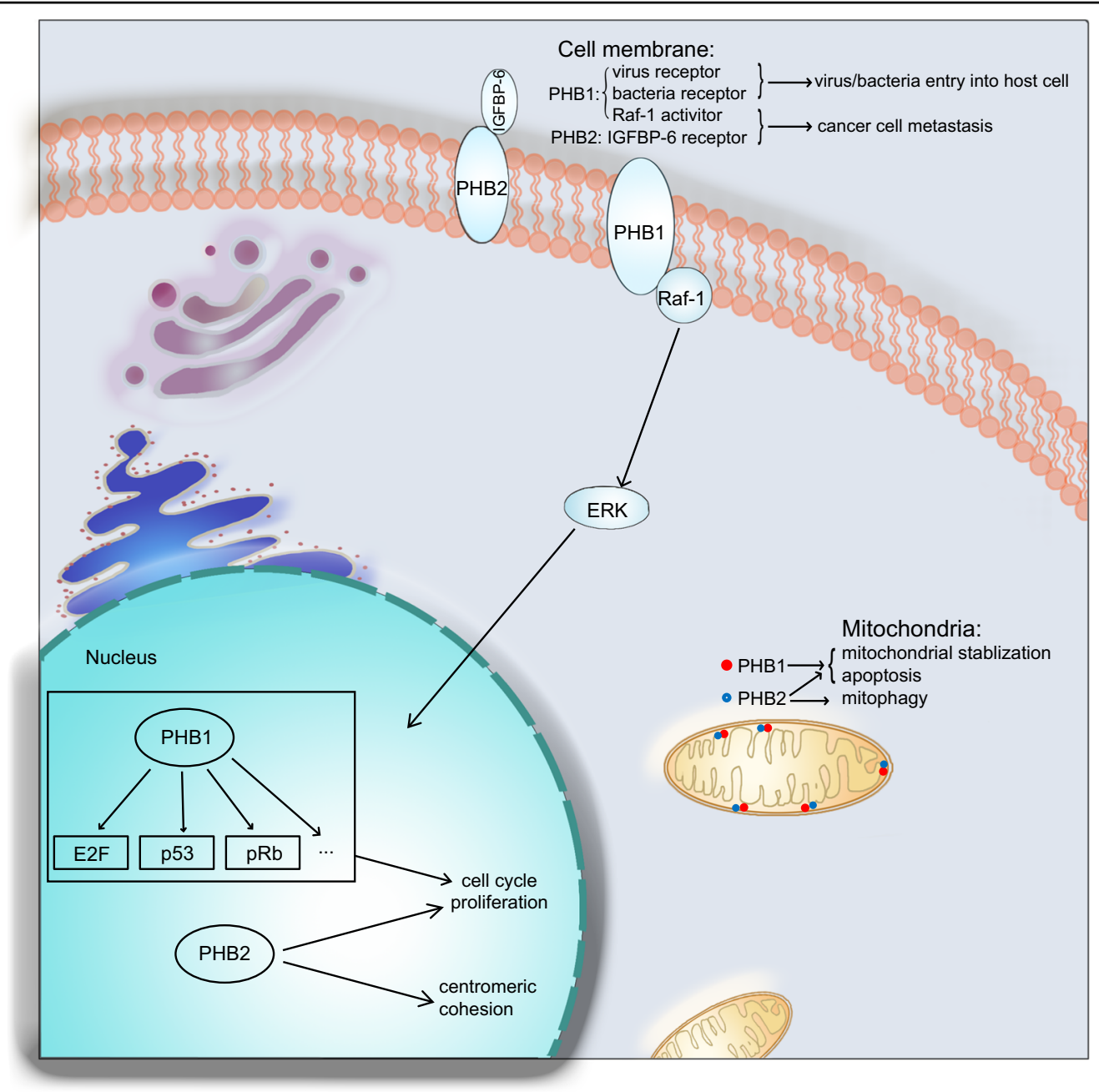

Fig. 1 Location and function of PHB1 and PHB2. PHB1 and PHB2 locate mainly in mitochondria inner membrane, nucleus and cell membrane. In mitochondria: PHB1 and PHB2 maintain mitochondrial stabilization and cell survival, and PHB2 also functions as a regulator in mitophagy. In nucleus: PHB1 binds to some transcription factors and play an important role in cell cycle process and cell proliferation, and PHB2 protects the centromeric cohesion during mitosis. In cell membrane: PHB1 not only acts as virus receptor to facilitate virus entry into host cells, but also interacts with Raf-1 and activates Raf-1/ERK signaling cascades to promote cancer cell metastasis, whereas PHB2 promotes cancer cell metastasis mainly through the interaction with IGFBP-6

key molecular regulator in the ER signaling pathway, inhibits the nuclear transportation of the PHB2/REA transcription complex, abolishing the inhibitory effect of the PHB2/REA complex on ER $\alpha$ transcriptional activity ${ }^{73}$, ${ }^{74}$. PHB2 was also shown to promote hepatocellular carcinoma growth and malignancy progression in the hypoxic tumor microenvironment ${ }^{75}$.

\section{Roles of PHB1 and PHB2 in cancer cell metastasis}

Despite many advances in the diagnosis and treatment of cancer, tumor metastasis remains largely incurable and up to $90 \%$ of cancer-related deaths are caused by metastatic disease rather than primary tumors ${ }^{76-78}$. Therefore, efficient biomarkers or targets involved in cancer metastasis are urgently needed to be identified. Interestingly, unlike its controversial role in cancer cell proliferation, PHB1 expression levels are significantly correlated with tumor metastasis and poor prognosis according to the current literature. In many cancers, the Raf-ERK signaling pathway is constitutively activated, promoting cancer cell metastasis. Recent studies have confirmed that PHB1 in lipid rafts is crucial for the activation of Raf- 1 and downstream signals. For example, studies in HeLa and CL1-0 cells showed that phosphorylation of PHB1 at T258 and Y259 is necessary for the activation of Raf-ERK signaling cascades supporting cancer cell metastasis ${ }^{14,} 79$. A similar mechanism was observed in pancreatic ductal adenocarcinoma and gallbladder cancer as well ${ }^{22,} 68$.

Some researches claimed that a polarized distribution of PHB1 in cells controls the migration direction of colorectal 


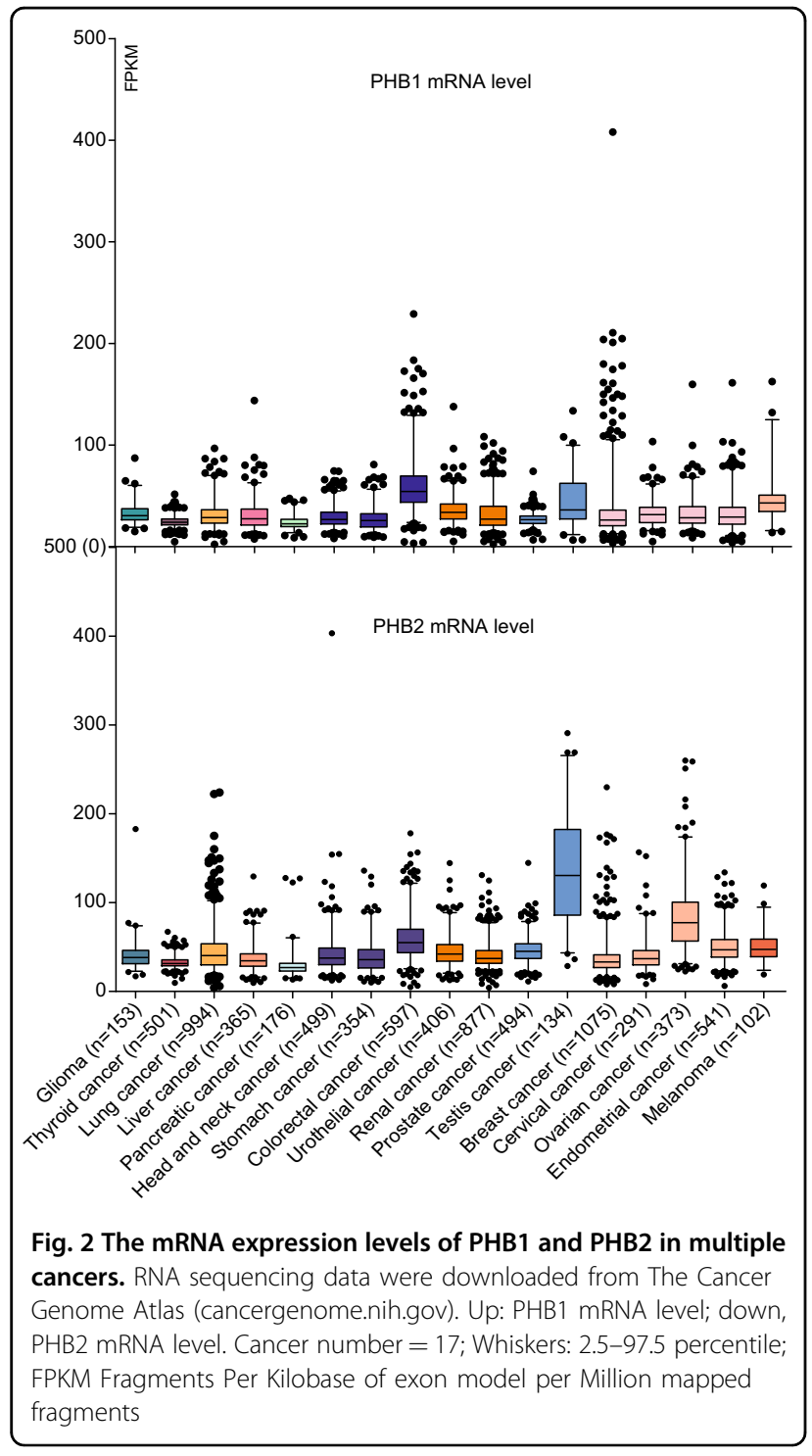

cancer cells. PHB1 can relocate to the luminal side of cells, face extracellular VEGF and indicate the direction of colorectal cancer cell invasion ${ }^{80}$. In lung cancer cells, studies verified that association of phospho-PHB1 T258 with MEKK1 activates the Snail, the repressor of E-cadherin, enhancing epithelial-mesenchymal transition (EMT) and lung cancer migration/invasion ${ }^{81,82}$. PHB1 overexpression is also reported in invasive breast carcinoma, indicating that PHB1 is a potential biomarker in breast cancer ${ }^{83}$, ${ }^{84}$. In prostate cancer, PHB2 was reported to interact with AKT2 and negatively regulate AKT2 expression, inducing cancer cell migration and malignancy ${ }^{49}$.

\section{Roles of PHB1 and PHB2 in cancer cell apoptosis}

Apoptosis is defined as programmed cell death that plays a fundamental role in organism development and tissue homeostasis. Disruption of apoptotic signaling

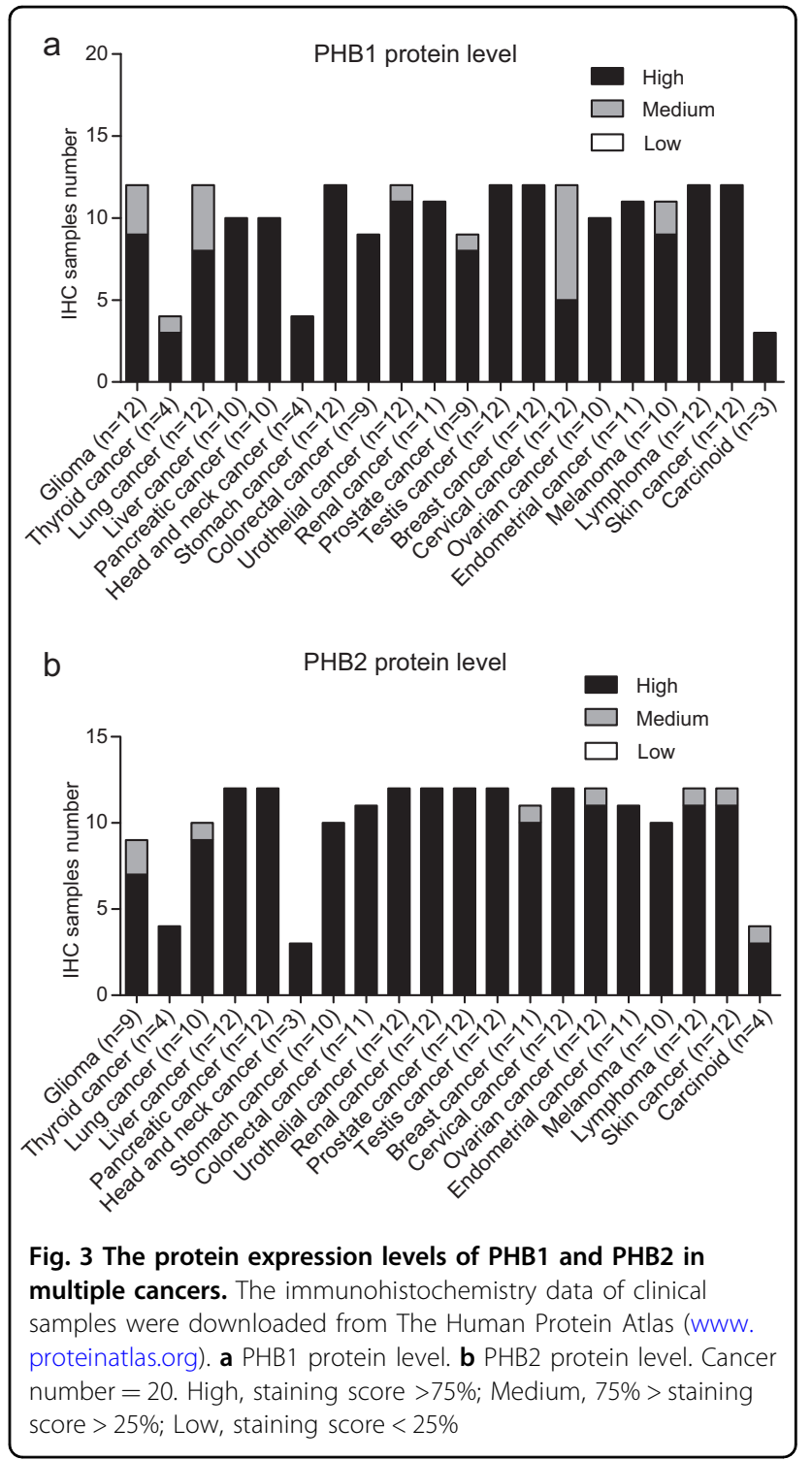

results in multiple diseases ${ }^{85-87}$. Apoptosis is also important for cancers to eliminate damaged cells that cannot be repaired, maintaining cancer cell vibrancy and aggressiveness $^{88}$. On the other hand, induction of apoptosis is a promising strategy for cancer treatment to eliminate cancer cells ${ }^{89-91}$. The role of PHB1 in cancer cell apoptosis has been explored in various studies. In breast and colon cancers, researchers demonstrated that PHB1 binds to the p53 induced gene 3 (PIG3) promoter motif (TGYCC) ${ }_{15}$ directly, promoting PIG3-mediated, p53-dependent cancer cell apoptosis ${ }^{92,93}$. Another study reported that cholesterol insufficiency in prostate cancer cells causes up-regulation of PHB1, inducing cell cycle arrest and apoptosis ${ }^{94}$. Furthermore, a series of experiments in colon cancer cells and melanoma cells confirmed that the direct interaction of PHB1 with trifluorothiazoline is necessary for trifluorothiazoline- 
induced apoptosis ${ }^{19}, 32$. This phenomenon was also observed in abrin-triggered apoptosis ${ }^{95}$ and the retinoic acid-resistant leukemia cell line NB4-R ${ }^{96}$. Interestingly, PHB1 expression significantly decreases, with its localization shifting from cytoplasmic to nuclear, and the colocalization of PHB1 with tumor suppressors such as p53 and $\mathrm{Rb}$ promotes apoptosis in cholangiocarcinoma ${ }^{97}$. Moreover, PHB2 can translocate from the mitochondria to the nucleus during capsaicin-induced apoptosis ${ }^{16}$. In ER-positive breast cancer, overexpression of PHB1 in MCF-7 and T47D cells significantly induces cell apoptosis $^{15}$, and the same phenomenon was detected in both gastric and liver cancer cells ${ }^{98,}{ }^{99}$.

In fact, PHB1 and PHB2 not only function in biological processes like cancer cell proliferation, metastasis and apoptosis in tumorigenesis and cancer development, but also play a role in modulating other processes such as cell differentiation in hepatocarcinoma, neuroblastoma and acute promyelocytic leukemia ${ }^{50,100,101}$ and mitophagy ${ }^{42}$. As the functional role of PHB1 and PHB2 in cancer becomes increasingly well defined, it is necessary to evaluate the upstream regulatory mechanisms of PHB1 and PHB2 in tumorigenesis to facilitate our understanding on the prohibitin domain family proteins and to generate clues for targeted drug development.

\section{Regulation of PHB1 and PHB2 in cancers}

Proteins in cells are usually regulated in three ways: transcriptional regulation ${ }^{102,} 103$, post-transcriptional regulation at the mRNA level ${ }^{104,} 105$ and protein modification ${ }^{106}$ including degradation ${ }^{107}$ and structural regulation $^{108}$. Transcriptional regulation mainly involves the regulation by transcription factors and epigenetic mechanisms ${ }^{109,}{ }^{110}$, while post-transcriptional regulation involves RNA interference ${ }^{111,112}$. Based on recent studies, all the three approaches of regulation seem to be involved in controlling prohibitin domain family proteins' effects on cancer cells.

\section{PHB1 is regulated at the transcriptional level}

At transcriptional level, researchers found that PHB1 expression level is associated with PHB1 genome copy number and a $3^{\prime}$ untranslated region (UTR) $1630 \mathrm{C}>\mathrm{T}$ polymorphism in gastric cancer ${ }^{113}$. Interestingly, the PHB1 genotype C1703T in the $3^{\prime}$-UTR is correlated with an increase in the risk for melanoma in a high ultraviolet radiation region in Brazil ${ }^{114}$. The expression of PHB1 was found to significantly increase in the thyroid tumor cells treated with trichostatin A (TSA) and sodium butyrate $(\mathrm{NaB})$, two histone deacetylase inhibitors, demonstrating that HDAC1/2 regulates both PHB1 transcription and alternative splicing ${ }^{115}$. Unfortunately, it remains unclear which transcription factors are responsible for PHB1 transcription.
PHB1 and PHB2 are regulated at the post-transcriptional level

At the post-transcriptional level, miR-27a targets the $3^{\prime}$-UTR of the PHB1 gene and down-regulates its expression in prostate cancer ${ }^{116}$. Likewise, the same mechanism has been confirmed in gastric adenocarcinoma cells and glioma cells ${ }^{117,}{ }^{118}$. Interestingly, miR-26a also binds directly to the $3^{\prime}$-UTR of $P H B 1$, inhibiting its expression in glioma cells ${ }^{66}$. In human melanoma cells, studies showed that miR-195 binds to the 3'-UTR of PHB1 as well ${ }^{119}$. Researchers also demonstrated that miR539 binds $P H B 2$ and suppresses its expression to induce mitochondrial fission and apoptosis ${ }^{120}$. Moreover, one study recently confirmed that a long noncoding RNA (lncRNA), prohibitin gene pseudogene 1 (PHBP1), which shares the high level of the nucleotide sequence identity (91.3\%) with its cognate gene $P H B 1$, promotes the stabilization of PHB1 mRNA by forming a PHBP1 RNA/PHB1 $m R N A$ heteroduplex in complementary regions, increasing the expression of $\mathrm{PHB}^{18}$.

\section{PHB1 is modified by AKT}

Protein modifications such as phosphorylation, glycosylation, ubiquitylation and small ubiquitin-related modifier (SUMO) determine the maturity, stability and activity of proteins ${ }^{121-123}$. For PHB1, phosphorylation or dephosphorylation at different amino acid sites results in different effects on tumorigenesis. Recent studies have focused on the phosphorylation of PHB1 at Tyr114, Ser121, Thr258, and Tyr $259^{124}$, with Thr258 and Tyr 259 phosphorylation well characterized in cancer cells. Phosphorylation of PHB1 at Thr258 in lipid rafts is necessary for the interaction of PHB1 with Raf-1. AKT phosphorylates PHB1 at Thr258, inducing the activation of Raf-1 and the Raf-1/ERK signaling pathway, resulting in cancer metastasis ${ }^{14,}{ }^{79}$. Interestingly, Tyr259 phosphorylation induces the activation of Thr258 and the downstream Raf-1/ERK signaling cascades.

Collectively, transcription of PHB1 is regulated by histone acetylation, microRNAs, and some lncRNAs. Moreover, phosphorylation of PHB1 at Thr258 and Tyr259 induced by AKT is important for cancer metastasis (Fig. 4a). Unfortunately, data on the upstream regulation of PHB2 is still lacking. Understanding of the regulation of prohibitin domain family and the related biological processes will offer us clues for further investigations on cancer diagnosis and treatment.

\section{Value of PHB1 and PHB2 as therapeutic targets in cancer treatment}

Many efficient targeted drugs have been developed for cancer treatment, balanced by increasing instances of clinical drug resistance ${ }^{125,126}$. Mechanistic studies found that most target genes or protein mutants develop 


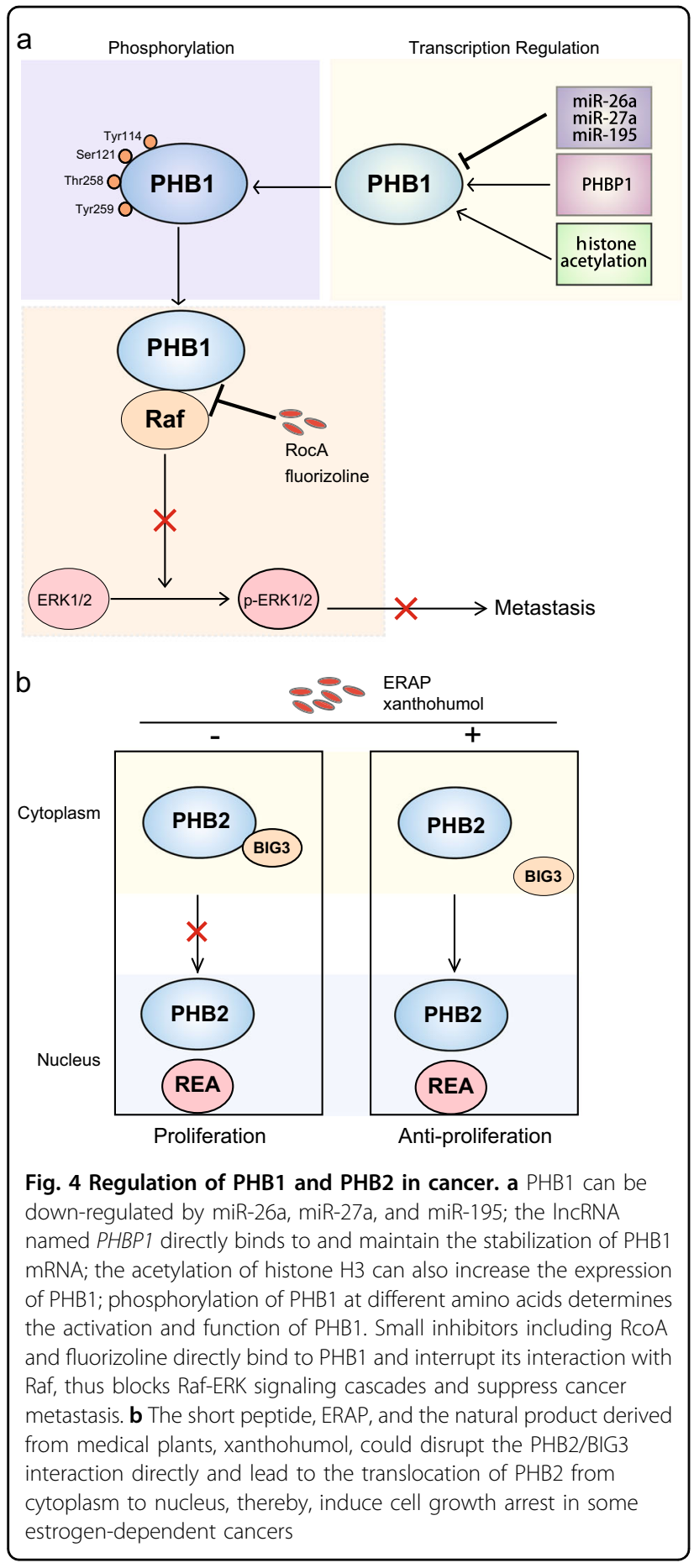

resistance to targeted drugs over a period of time ${ }^{127-129}$. Thus, new effective targets are always needed. As summarized above, PHB1 acts as a tumor promoter in most cancer types, and thus is a potential target for novel drug development, especially the PHB1 located in the cell membrane. In fact, several small inhibitors targeting PHB1 or PHB1/Raf-1 or PHB2 interactions have been identified, and tumors were significantly inhibited by these drugs.

\section{Drugs targeting PHB1}

Rocaglamide (RocA), a naturally occurring compound isolated from medicinal plants belonging to the genus Aglaia family Meliaceae ${ }^{20}$, was used for treatment of coughs, injuries, asthma, and inflammatory skin diseases. RocA directly targets $\mathrm{PHB1}$ and prevents its interaction with Raf-1, resulting in impaired ERK1/2 activation in leukemic cells ${ }^{130}$. The effect of RocA was further displayed in pancreatic ductal adenocarcinoma, where RocA inhibits the interaction of PHB1 and Raf-1, significantly suppressing cancer cell growth and metastasis ${ }^{22}$. Interestingly, RocA can reverse Raf-1-dependent resistance to vemurafenib, inhibite cell growth and induce apoptosis in melanoma cells $^{23}$, indicating that RocA has potential value for use in vemurafenib-resistant cancers.

Recently, researchers demonstrated that a small molecular inhibitor fluorizoline, synthetized based on natural products from medical plants, also prevents Ras-Raf interaction in lung cancer cell and inhibits tumor growth and metastasis ${ }^{131}$. Moreover, fluorizoline induces mitochondrial-dependent apoptosis by targeting at PHB1 in a p53-independent manner in MEF cells, as well as in multiple cancers ${ }^{19}, 33,132$. The combined use of RocA and fluorizoline may be a promising strategy for cancer treatment (Fig. 4a).

\section{Drugs targeting PHB2}

As PHB2 is involved in estrogen-dependent cancers such as breast cancer, and BIG3 promotes cancer cell proliferation by inhibiting PHB2 nuclear translocation and promoting the activity of ER $\alpha$, the BIG3/PHB2 interaction is necessary for tumorigenesis ${ }^{73,74}$. Therefore, inhibition of the interaction of BIG3-PHB2 is a feasible strategy in estrogen-dependent cancer therapy. In fact, researchers isolated the stable ER $\alpha$ activity-regulator synthetic peptide (ERAP: 165-177 amino acids), a short peptide derived from $\alpha$-helical BIG3 sequence that specifically binds to PHB2 and competitively prevents the BIG3/PHB2 interaction, and then showed that ERAP promotes PHB2/REA complex nuclear translocation and suppresses cancer cell proliferation in vitro and in vivo ${ }^{133}$. Moreover, PHB2 released from the BIG3-PHB2 complex by ERAP treatment reduces phosphorylation levels of AKT and MAPK, resulting in significant suppression of proliferation in ER $\alpha$-positive breast cancer cells. More importantly, the tumor suppressive effects of ERAP are enhanced by combined use with tamoxifen, indicating that ERAP treatment can reverse tamoxifen resistance and enhance tamoxifen responsiveness in ER $\alpha$-positive breast cancer cells ${ }^{24,25}$. The same research team also identified a natural product derived from medical plants named xanthohumol that could interrupt BIG3/PHB2 interaction and suppress ER $\alpha$-positive breast cancer cell proliferation (Fig. $4 \mathrm{~b})^{134}$. 
New target exploration is a continuous effort for new drug development, offering more opportunities to overcome drug resistance in cancer treatment. Based on the special localization and clear mechanism of PHB1 in tumorigenesis, there is a bright future for developing high-efficiency drugs to target PHB1 for cancer treatment. PHB2 inhibitors will also play an important role in estrogen-dependent cancer treatment, especially in premenopausal women.

\section{Conclusion and future perspectives}

Since the $P H B$ gene was first isolated and characterized in rat liver in 1989, researchers have been studying this "anti-proliferation" gene to elucidate its role in biological process and diseases.

Biomarkers and genes for targeted drug development are important for cancer diagnosis and treatment, and drugs developed based on proteins such as EGFR have been extensively used for clinical treatment ${ }^{135}$. However, increasing numbers of patients are presenting with drug resistance when treated with extant drugs for long periods of time ${ }^{128}$. Thus, exploration of new targets is urgently needed. Differential PHB1 expression has been identified in multiple cancers as compared with normal tissues; hence, the PHB1 protein, especially when located in the cell membrane, may be a perfect choice for targeted drug development, as drugs could target PHB1 directly without transportation into cells. The observations that small molecule inhibitors RocA and fluorizoline target PHB1, and a short peptide ERAP and xanthohumol target PHB2, provide us stimulation in anti-cancer drug design. At least, modifying these existing drugs to increase their efficiency, or screening more potent inhibitors targeting PHB1 and PHB2 may lead to breakthroughs in cancer therapy.

\section{Acknowledgements}

We thank Qihua Zhang for the artwork preparation. This work was supported by the National Key Research and Development Program of China (2017YFA0505100/2017YFA0505000), the National Natural Science Foundation of China (31770888, 31570828, 81773085), and Guangdong Natural Science Research Grant (\$2013030013315, 2016A030313838).

\section{Conflict of interest}

The authors declare that they have no conflict of interest.

\section{Publisher's note}

Springer Nature remains neutral with regard to jurisdictional claims in published maps and institutional affiliations.

Received: 15 March 2018 Revised: 27 April 2018 Accepted: 3 May 2018 Published online: 21 May 2018

\section{References}

1. McClung, J. K. et al. Isolation of a CDNA that hybrid selects antiproliferative mRNA from rat liver. Biochem. Biophys. Res. Commun. 164, 1316-1322 (1989).
2. Sato, $T$. et al. The human prohibitin (PHB) gene family and its somatic mutations in human tumors. Genomics 17, 762-764 (1993).

3. Sato, T. et al. The human prohibitin gene located on chromosome $17 \mathrm{q} 21$ is mutated in sporadic breast cancer. Cancer Res. 52, 1643-1646 (1992).

4. White, J. J. et al. Assignment of the human prohibitin gene (PHB) to chromosome 17 and identification of a DNA polymorphism. Genomics 11, 228-230 (1991).

5. Mishra, S., Murphy, L. C., Nyomba, B. L. \& Murphy, L. J. Prohibitin: a potential target for new therapeutics. Trends Mol. Med. 11, 192-197 (2005).

6. Nijtmans, L. G. et al. Prohibitins act as a membrane-bound chaperone for the stabilization of mitochondrial proteins. EMBO J. 19, 2444-2451 (2000).

7. Tatsuta, T. \& Langer, T. Quality control of mitochondria: protection against neurodegeneration and ageing. EMBO J. 27, 306-14.14 (2008).

8. Wintachai, P. et al. Identification of prohibitin as a Chikungunya virus receptor protein. J. Med. Virol. 84, 1757-1770 (2012).

9. Desideri, E., Cavallo, A. L. \& Baccarini, M. Alike but different: RAF paralogs and their signaling outputs. Cell 161, 967-970 (2015).

10. Fu, P., Yang, Z. \& Bach, L. A. Prohibitin-2 binding modulates insulin-like growth factor-binding protein-6 (IGFBP-6)-induced rhabdomyosarcoma cell migration. J. Biol. Chem. 288, 29890-29900 (2013).

11. Chowdhury, l., Thomas, K. \& Thompson, W. E. Prohibitin(PHB) roles in granulosa cell physiology. Cell Tissue Res. 363, 19-29 (2016).

12. Koushyar, S. et al. The prohibitin-repressive interaction with E2F1 is rapidly inhibited by androgen signalling in prostate cancer cells. Oncogenesis $\mathbf{6}$, e333 (2017).

13. Takata, H. et al. PHB2 protects sister-chromatid cohesion in mitosis. Curr. Biol. 17, 1356-1361 (2007).

14. Chiu, C. F. et al. Raf activation by Ras and promotion of cellular metastasis require phosphorylation of prohibitin in the raft domain of the plasma membrane. Oncogene 32, 777-787 (2013).

15. Liu, P. et al. Prohibitin promotes androgen receptor activation in ER-positive breast cancer. Cell Cycle 16, 776-784 (2017).

16. Kuramori, C. et al. Capsaicin binds to prohibitin 2 and displaces it from the mitochondria to the nucleus. Biochem. Biophys. Res. Commun. 379, 519-525 (2009).

17. Liao, Q. et al. Prohibitin is an important biomarker for nasopharyngeal carcinoma progression and prognosis. Eur. J. Cancer Prev. 22, 68-76 (2013).

18. Feng, F., Qiu, B., Zang, R., Song, P. \& Gao, S. Pseudogene PHBP1 promotes esophageal squamous cell carcinoma proliferation by increasing its cognate gene PHB expression. Oncotarget 8, 29091-29100 (2017).

19. Pérez-Perarnau, A. et al. A trifluorinated thiazoline scaffold leading to proapoptotic agents targeting prohibitins. Angew. Chem. Int. Ed. Engl. 53, 10150-10154 (2014).

20. Kim, S., Salim, A. A., Swanson, S. M. \& Kinghorn, A. D. Potential of cyclopenta [b]benzofurans from Aglaia species in cancer chemotherapy. Anticancer Agents Med. Chem. 6, 319-345 (2006).

21. Ebada, S. S., Lajkiewicz, N., Porco, J. A. Jr, Li-Weber, M. \& Proksch, P. Chemistry and biology of rocaglamides (=flavaglines) and related derivatives from aglaia species (meliaceae). Prog. Chem. Org. Nat. Prod. 94, 1-58 (2011).

22. Luan, Z., He, Y., Alattar, M., Chen, Z. \& He, F. Targeting the prohibitin scaffoldCRAF kinase interaction in RAS-ERK-driven pancreatic ductal adenocarcinoma. Mol. Cancer 13, 38 (2014).

23. Doudican, N. A. \& Orlow, S. J. Inhibition of the CRAF/prohibitin interaction reverses CRAF-dependent resistance to vemurafenib. Oncogene 36, 423-428 (2017).

24. Yoshimaru, T. et al. Targeting BIG3-PHB2 interaction to overcome tamoxifen resistance in breast cancer cells. Nat. Commun. 4, 2443 (2013).

25. Yoshimaru, T. et al. Therapeutic advances in BIG3-PHB2 inhibition targeting the crosstalk between estrogen and growth factors in breast cancer. Cancer Sci. 106, 550-558 (2015).

26. Morrow, I. C. et al. Flotillin-1/reggie-2 traffics to surface raft domains via a novel golgi-independent pathway. J. Biol. Chem. 277, 48834-48841 (2002).

27. Bavelloni, A., Piazzi, M., Raffini, M., Faenza, I. \& Blalock, W. L. Prohibitin 2: At a communications crossroads. IUBMB Life 67, 239-254 (2015).

28. Back, J. W. et al. A structure for the yeast prohibitin complex: structure prediction and evidence from chemical crosslinking and mass spectrometry. Protein Sci. 11, 2471-2478 (2002).

29. López-Huertas, M. R. et al. The presence of HIV-1 Tat protein second exon delays fas protein-mediated apoptosis in CD4 + T lymphocytes: a potential mechanism for persistent viral production. J. Biol. Chem. 288, 7626-7644 (2013). 
30. Artal-Sanz, M. \& Tavernarakis, N. Prohibitin and mitochondrial biology. Trends Endocrinol. Metab. 20, 394-401 (2009).

31. Ising, C. \& Brinkkoetter, P. T. Prohibitin signaling at the kidney filtration barrier Adv. Exp. Med. Biol. 982, 563-575 (2017).

32. Bollu, L. R. et al. Involvement of de novo synthesized palmitate and mitochondrial EGFR in EGF induced mitochondrial fusion of cancer cells. Cell Cycle 13, 2415-2430 (2014).

33. Moncunill-Massaguer, C. et al. A novel prohibitin-binding compound induces the mitochondrial apoptotic pathway through NOXA and BIM upregulation. Oncotarget 6, 41750-41765 (2015).

34. Yang, H. B. et al. Differential expression and regulation of prohibitin during curcumin-induced apoptosis of immortalized human epidermal HaCaT cells. Int. J. Mol. Med. 33, 507-514 (2014).

35. Ising, C. et al. Prohibitin-2 depletion unravels extra-mitochondrial functions at the kidney filtration barrier. Am. J. Pathol. 186, 1128-1139 (2016).

36. Lachén-Montes, M. et al. Olfactory bulb neuroproteomics reveals a chronological perturbation of survival routes and a disruption of prohibitin complex during Alzheimer's disease progression. Sci. Rep. 7, 9115 (2017).

37. Merkwirth, $\mathrm{C}$. et al. Loss of prohibitin membrane scaffolds impairs mitochondrial architecture and leads to tau hyperphosphorylation and neurodegeneration. PLoS Genet. 8, e1003021 (2012).

38. Kahl, A. et al. Neuronal expression of the mitochondrial protein prohibitin confers profound neuroprotection in a mouse model of focal cerebral ischemia. J. Cereb. Blood Flow Metab. 2017; https://doi.org/10.1177/ $0271678 \times 17720371$.

39. Zhou, P. et al. Prohibitin reduces mitochondrial free radical production and protects brain cells from different injury modalities. J. Neurosci. 32, 583-592 (2012).

40. Dutta, D., et al. Low levels of prohibitin in substantia nigra makes dopaminergic neurons vulnerable in Parkinson's disease. Mol. Neurobiol. 2017; https://doi.org/10.1007/s12035-016-0328-y.

41. Chai, R. R. et al. Prohibitin involvement in the generation of mitochondrial superoxide at complex I in human sperm. J. Cell Mol. Med. 21, 121-129 (2017).

42. Wei, Y., Chiang, W. C., Sumpter, R. Jr, Mishra, P. \& Levine, B. Prohibitin 2 is an inner mitochondrial membrane mitophagy receptor. Cell 168, 224-238 (2017).

43. Toska, E., Shandilya, J., Goodfellow, S. J., Medler, K. F. \& Roberts, S. G. Prohibitin is required for transcriptional repression by the WT1-BASP1 complex. Oncogene 33, 5100-5108 (2014).

44. Gamble, S. C. et al. Androgens target prohibitin to regulate proliferation of prostate cancer cells. Oncogene 23, 2996-3004 (2004).

45. Wang, S., Nath, N., Adlam, M. \& Chellappan, S. Prohibitin, a potential tumor suppressor, interacts with RB and regulates E2F function. Oncogene $\mathbf{1 8}$ 3501-3510 (1999).

46. He, B. et al. A repressive role for prohibitin in estrogen signaling. Mol. Endocrinol. 22, 344-360 (2008).

47. Dong, P. et al. Induction of paclitaxel resistance by ERa mediated prohibitin mitochondrial-nuclear shuttling. PLoS ONE 8, e83519 (2013).

48. Teittinen, K. J. et al. Nucleolar proteins with altered expression in leukemic cell lines. Leuk. Res. 36, 232-236 (2012).

49. Shen, Y. et al. Prohibitin-2 negatively regulates AKT2 expression to promote prostate cancer cell migration. Int. J. Mol. Med. 41, 1147-1155 (2018).

50. Bavelloni, A. et al. Prohibitin 2 represents a novel nuclear AKT substrate during all-trans retinoic acid-induced differentiation of acute promyelocytic leukemia cells. FASEB J. 28, 2009-2019 (2014).

51. Knopf, J. D. et al. The stromal cell-surface protease fibroblast activation protein-a localizes to lipid rafts and is recruited to invadopodia. Biochim. Biophys. Acta 1853(10 Pt A), 2515-2525 (2015).

52. Zhou, T. B. \& Qin, Y. H. Signaling pathways of prohibitin and its role in diseases. J. Recept. Signal. Transduct. Res. 33, 28-36 (2013).

53. Peng, Y. T., Chen, P., Ouyang, R. Y. \& Song, L. Multifaceted role of prohibitin in cell survival and apoptosis. Apoptosis 20, 1135-1149 (2015).

54. Liu, C. et al. Identification of human host proteins contributing to H5N1 influenza virus propagation by membrane proteomics. J. Proteome Res. 11, 5396-5405 (2012).

55. Paingankar, M. S., Gokhale, M. D. \& Deobagkar, D. N. Dengue-2-virusinteracting polypeptides involved in mosquito cell infection. Arch. Virol. 155, 1453-1461 (2010).

56. Yurugi, $\mathrm{H}$. et al. Expression of prohibitins on the surface of activated $\mathrm{T}$ cells. Biochem. Biophys. Res. Commun. 420, 275-280 (2012).
57. Santhanam, S. K., Dutta, D., Parween, F. \& Qadri, A. The virulence polysaccharide $V i$ released by Salmonella Typhi targets membrane prohibitin to inhibit T-cell activation. J. Infect. Dis. 210, 79-88 (2014).

58. Sharma, A. \& Qadri, A. Vi polysaccharide of Salmonella typhi targets the prohibitin family of molecules in intestinal epithelial cells and suppresses early inflammatory responses. Proc. Natl Acad. Sci. USA 101, 17492-17497 (2004).

59. Zhong, N., Cui, Y., Zhou, X., Li, T. \& Han, J. Identification of prohibitin 1 as a potential prognostic biomarker in human pancreatic carcinoma using modified aqueous two-phase partition system combined with 2D-MALDITOF-TOF-MS/MS. Tumour Biol. 36, 1221-1231 (2015).

60. Zhang, Y., Wang, Y., Xiang, Y., Lee, W. \& Zhang, Y. Prohibitins are involved in protease-activated receptor 1-mediated platelet aggregation. J. Thromb. Haemost. 10, 411-418 (2012).

61. Wang, Y. J. et al. Prohibitin is involved in the activated internalization and degradation of protease-activated receptor 1. Biochim. Biophys. Acta 1843, 1393-1401 (2014).

62. Uhlen, M. et al. A pathology atlas of the human cancer transcriptome. Science 357, eaan2507 (2017).

63. Zhang, W. et al. Skp2 is over-expressed in breast cancer and promotes breast cancer cell proliferation. Cell Cycle 15, 1344-1351 (2016).

64. Du, M. D., He, K. Y., Qin, G., Chen, J. \& Li, J. Y. Adriamycin resistance-associated prohibitin gene inhibits proliferation of human osteosarcoma MG63 cells by interacting with oncogenes and tumor suppressor genes. Oncol. Lett. 12, 1994-2000 (2016).

65. Chumbalkar, V. C. et al. Differential protein expression in human gliomas and molecular insights. Proteomics 5, 1167-1177 (2005).

66. Qian, X. et al. MicroRNA-26a promotes tumor growth and angiogenesis in glioma by directly targeting prohibitin. CNS Neurosci. Ther. 19, 804-812 (2013).

67. Lu, J. J. et al. Proteomic analysis of hepatocellular carcinoma HepG2 cells treated with platycodin D. Chin. J. Nat. Med 13, 673-679 (2015).

68. Cao, Y. et al. Prohibitin overexpression predicts poor prognosis and promotes cell proliferation and invasion through ERK pathway activation in gallbladder cancer. J. Exp. Clin. Cancer Res. 35, 68 (2016).

69. Jiang, L. et al. Akt phosphorylates Prohibitin 1 to mediate its mitochondrial localization and promote proliferation of bladder cancer cells. Cell Death Dis. 6, e1660 (2015)

70. $\mathrm{Wu}, \mathrm{T}$. F. et al. Prohibitin in the pathogenesis of transitional cell bladder cancer. Anticancer Res. 27, 895-900 (2007).

71. El-Etreby, N. M., Ghazy, A. A. \& Rashad, R. Prohibitin: targeting peptide coupled to ovarian cancer, luteinization and TGF- $\beta$ pathways. J. Ovarian Res. 10 28 (2017).

72. Dai, Z. et al. Mitochondrial comparative proteomics of human ovarian cancer cells and their platinum-resistant sublines. Proteomics 10, 3789-3799 (2010).

73. Nakamura, A., Osonoi, T. \& Terauchi, Y. Relationship between urinary sodium excretion and pioglitazone-induced edema. J. Diabetes Investig. 1, 208-211 (2010)

74. Kim, N. H. et al. BIG3 inhibits the estrogen-dependent nuclear translocation of PHB2 via multiple karyopherin-alpha proteins in breast cancer cells. PLoS ONE 10, e0127707 (2015)

75. Cheng, J. et al. Prohibitin-2 promotes hepatocellular carcinoma malignancy progression in hypoxia based on a label-free quantitative proteomics strategy. Mol. Carcinog. 53, 820-832 (2014).

76. Turajic, S. \& Swanton, C. Metastasis as an evolutionary process. Science $\mathbf{3 5 2}$ 169-175 (2016).

77. Lambert, A. W., Pattabiraman, D. R. \& Weinberg, R. A. Emerging biological principles of metastasis. Cell 168, 670-691 (2017)

78. Massagué, J. \& Obenauf, A. C. Metastatic colonization by circulating tumour cells. Nature 529, 298-306 (2016)

79. Chiu, C. F., Peng, J. M., Hung, S. W., Liang, C. M. \& Liang, S. M. Recombinant viral capsid protein VP1 suppresses migration and invasion of human cervica cancer by modulating phosphorylated prohibitin in lipid rafts. Cancer Lett. 320, 205-214 (2012).

80. $\mathrm{Ma}$, L. L. et al. Prohibitin, relocated to the front ends, can control the migration directionality of colorectal cancer cells. Oncotarget 8, 76340-76356 (2017).

81. Ho, M. Y., Liang, C. M. \& Liang, S. M. MIG-7 and phosphorylated prohibitin coordinately regulate lung cancer invasion/metastasis. Oncotarget 6, 381-393 (2015).

82. Wong, P. F. et al. Eurycomanone suppresses expression of lung cancer cell tumor markers, prohibitin, annexin 1 and endoplasmic reticulum protein 28 Phytomedicine 19, 138-144 (2012). 
83. Canevari, R. A. et al. Identification of novel biomarkers associated with poor patient outcomes in invasive breast carcinoma. Tumour Biol. 37, 13855-13870 (2016).

84. Koushyar, S., Jiang, W. G. \& Dart, D. A. Unveiling the potential of prohibitin in cancer. Cancer Lett. 369, 316-322 (2015).

85. Fuchs, Y. \& Steller, H. Programmed cell death in animal development and disease. Cell 147, 742-758 (2011).

86. Green, D. R., Galluzzi, L. \& Kroemer, G. Cell biology. Metabolic control of cell death. Science 345, 1250256 (2014).

87. Bredesen, D. E., Rao, R. V. \& Mehlen, P. Cell death in the nervous system. Nature 443, 796-802 (2006).

88. Evan, Gerardl. \& Vousden, KarenH. Proliferation, cell cycle and apoptosis in cancer. Nature 411, 342-348 (2001).

89. Lowe, ScottW., Cepero, Enrique \& Evan, Gerard Intrinsic tumour suppression. Nature 432, 307-315 (2004).

90. Croce, C. M. \& Reed, J. C. Finally, an apoptosis-targeting therapeutic for cancer. Cancer Res. 76, 5914-5920 (2016).

91. Igea, A. \& Nebreda, A. R. The Stress Kinase p38a as a Target for Cancer Therapy. Cancer Res. 75, 3997-4002 (2015).

92. Guan, X., Liu, Z., Wang, L., Johnson, D. G. \& Wei, Q. Identification of prohibitin and prohibiton as novel factors binding to the p53 induced gene 3 (PIG3) promoter (TGYCC)(15) motif. Biochem. Biophys. Res. Commun. 443 1239-1244 (2014).

93. Zhang, W. et al. BRCA1 regulates PIG3-mediated apoptosis in a p53dependent manner. Oncotarget 6, 7608-7618 (2015).

94. Dong, P. Flores, J., Pelton, K. \& Solomon, K. R. Prohibitin is a cholesterolsensitive regulator of cell cycle transit. J. Cell. Biochem. 111, 1367-1374 (2010).

95. Liu, Y. H., Peck, K. \& Lin, J. Y. Involvement of prohibitin upregulation in abrintriggered apoptosis. Evid. Based Complement. Altern. Med. 2012, 605154 (2012).

96. Liu, Y., He, P., Zhang, M. \& Wu, D. Lentiviral vector-mediated RNA interference targeted against prohibitin inhibits apoptosis of the retinoic acid-resistant acute promyelocytic leukemia cell line NB4-R1. Mol. Med. Rep. 6, 1288-1292 (2012)

97. Song, W., Tian, L., Li, S. S., Shen, D. Y. \& Chen, Q. X. The aberrant expression and localization of prohibitin during apoptosis of human cholangiocarcinoma Mz-ChA-1 cells. FEBS Lett. 588, 422-428 (2014).

98. Zhang, L., Ji, Q., Ni, Z. H. \& Sun, J. Prohibitin induces apoptosis in BGC823 gastric cancer cells through the mitochondrial pathway. Asian Pac. J. Cancer Prev. 13, 3803-3807 (2012)

99. Yoo, D. R. et al. Proteomic identification of anti-cancer proteins in luteolintreated human hepatoma Huh-7 cells. Cancer Lett. 282, 48-54 (2009).

100. $\mathrm{Xu}, \mathrm{D}$. H. et al. Positional and expressive alteration of prohibitin during the induced differentiation of human hepatocarcinoma SMMC-7721 cells. World J. Gastroenterol. 14, 5008-5014 (2008).

101. Li, Q. F. et al. Localization of prohibitin in the nuclear matrix and alteration of its expression during differentiation of human neuroblastoma SK-N-SH cells induced by retinoic acid. Cell. Mol. Neurobiol. 31, 203-211 (2011).

102. Hnisz, D., Shrinivas, K., Young, R. A., Chakraborty, A. K. \& Sharp, P. A. A Phase Separation Model for Transcriptional Control. Cell 169, 13-23 (2017).

103. Bradner, J. E., Hnisz, D. \& Young, R. A. Transcriptional addiction in cancer. Cell 168, 629-643 (2017).

104. Popovitchenko, T. \& Rasin, M. R. Transcriptional and post-transcriptional mechanisms of the development of neocortical lamination. Front. Neuroanat. 11, 102 (2017).

105. Liu, W., Ma, R. \& Yuan, Y. Post-transcriptional Regulation of Genes Related to Biological Behaviors of Gastric Cancer by Long Noncoding RNAs and MicroRNAs. J. Cancer 8, 4141-4154 (2017).

106. Liang, Z. et al. SUMOylation of IQGAP1 promotes the development of colorectal cancer. Cancer Lett. 411, 90-99 (2017).

107. Goldberg, A. L. Protein degradation and protection against misfolded or damaged proteins. Nature 426, 895-899 (2003).

108. Wang, M. \& Kaufman, R. J. Protein misfolding in the endoplasmic reticulum as a conduit to human disease. Nature 529, 326-335 (2016).

109. Karin, M. Nuclear factor-kappaB in cancer development and progression. Nature 441, 431-436 (2006).
110. Schübeler, D. Function and information content of DNA methylation. Nature 517, 321-326 (2015)

111. Chitwood, D. H. \& Timmermans, M. C. Small RNAs are on the move. Nature 467, 415-419 (2010)

112. Castanotto, D. \& Rossi, J. J. The promises and pitfalls of RNA-interferencebased therapeutics. Nature 457, 426-433 (2009).

113. Leal, M. F. et al. Prohibitin expression deregulation in gastric cancer is associated with the $3^{\prime}$ untranslated region 1630 C T polymorphism and copy number variation. PLOS ONE 9, e98583 (2014).

114. Francisco, G. et al. Polymorphisms in the p27kip-1 and prohibitin genes denote novel genes associated with melanoma risk in Brazil, a high ultraviolet index region. Melanoma Res. 23, 231-236 (2013).

115. Puppin, C., Passon, N., Franzoni, A., Russo, D. \& Damante, G. Histone deacetylase inhibitors control the transcription and alternative splicing of prohibitin in thyroid tumor cells. Oncol. Rep. 25, 393-397 (2011).

116. Fletcher, C. E. et al. Androgen-regulated processing of the oncomir miR-27a which targets Prohibitin in prostate cancer. Hum. Mol. Genet. 21, 3112-3127 (2012)

117. Liu, T., Tang, H., Lang, Y., Liu, M. \& Li, X. MicroRNA-27a functions as an oncogene in gastric adenocarcinoma by targeting prohibitin. Cancer Lett 273, 233-242 (2009).

118. Chen, W. et al. Emerging role of microRNA-27a in human malignant glioma cell survival via targeting of prohibitin. Mol. Med. Rep. 12, 1515-1523 (2015).

119. Cirilo, P. D. R. et al. MicroRNA-195 acts as an anti-proliferative miRNA in human melanoma cells by targeting Prohibitin 1. BMC Cancer 17, 750 (2017).

120. Wang, $K$. et al. CARL IncRNA inhibits anoxia-induced mitochondrial fission and apoptosis in cardiomyocytes by impairing miR-539-dependent PHB2 downregulation. Nat. Commun. 5, 3596 (2014).

121. Frankson, R. et al. Therapeutic targeting of oncogenic tyrosine phosphatases. Cancer Res. 77, 5701-5705 (2017).

122. Ohtsubo, K. \& Marth, J. D. Glycosylation in cellular mechanisms of health and disease. Cell 126, 855-867 (2006).

123. Bergink, S. \& Jentsch, S. Principles of ubiquitin and SUMO modifications in DNA repair. Nature 458, 461-467 (2009).

124. Ande, S. R. Moulik, S. \& Mishra, S. Interaction between O-GlcNAc modification and tyrosine phosphorylation of prohibitin: implication for a novel binary switch. PLOS ONE 4, e4586 (2009).

125. Ruffell, B. \& Coussens, L. M. Macrophages and therapeutic resistance in cancer. Cancer Cell. 27, 462-472 (2015).

126. Wood, K. C. Mapping the pathways of resistance to targeted therapies Cancer Res. 75, 4247-4251 (2015).

127. Ramos, P. \& Bentires-Alj, M. Mechanism-based cancer therapy: resistance to therapy, therapy for resistance. Oncogene 34, 3617-3626 (2014).

128. Chong, C. R. \& Jänne, P. A. The quest to overcome resistance to EGFRtargeted therapies in cancer. Nat. Med. 19, 1389-1400 (2013).

129. Lord, C. J. SpringerAmpamp; Ashworth, A. Mechanisms of resistance to therapies targeting BRCA-mutant cancers. Nat. Med. 19, 1381-1388 (2013).

130. Polier, G. et al. The natural anticancer compounds rocaglamides inhibit the Raf-MEK-ERK pathway by targeting prohibitin 1 and 2. Chem. Biol. 19 1093-1104 (2012).

131. Yurugi, $H$. et al. Targeting prohibitins with chemical ligands inhibits KRAS mediated lung tumours. Oncogene 36, 4778-4789 (2017).

132. Cosialls, A. M. et al. The prohibitin-binding compound fluorizoline induces apoptosis in chronic lymphocytic leukemia cells through the upregulation of NOXA and synergizes with ibrutinib, 5-aminoimidazole-4-carboxamide riboside or venetoclax. Haematologica 102, 1587-1593 (2017).

133. Yoshimaru, T. et al. Stapled BIG3 helical peptide ERAP potentiates antitumour activity for breast cancer therapeutics. Sci. Rep. 7, 1821 (2017).

134. Yoshimaru, T. et al. Xanthohumol suppresses oestrogen-signalling in breast cancer through the inhibition of BIG3-PHB2 interactions. Sci. Rep. 4, 7355 (2014).

135. Gan, H. K., Burgess, A. W., Clayton, A. H. \& Scott, A. M. Targeting of a conformationally exposed, tumor-specific epitope of EGFR as a strategy for cancer therapy. Cancer Res. 72, 2924-2930 (2012). 